Europhysics Letters

PREPRINT

\title{
Clustering in gravitating N-body systems
}

\author{
M. Bottaccio ${ }^{1,2}$, A. Amici ${ }^{1}$, P. Miocchi ${ }^{1}$, R. Capuzzo Dolcetta ${ }^{1}$, M. Mon- \\ TUOrI $^{2}$ and L. Pietronero ${ }^{1,2}$ \\ 1 Physics Department, University of Rome "La Sapienza", Italy, \\ 2 INFM, Research Unit of Roma "La Sapienza", Italy.
}

PACS. 05.20.-y - Classical statistical mechanics.

PACS. 45.50.Jf - Few- and many-body systems.

PACS. 45.50.-j - Dynamics and kinematics of a particle and a system of particles.

\begin{abstract}
We study gravitational clustering of mass points in three dimensions with random initial positions and periodic boundary conditions (no expansion) by numerical simulations. Correlation properties are well defined in the system and a sort of thermodynamic limit can be defined for the transient regime of clustering. Structure formation proceeds along two paths: (i) fluid-like evolution of density perturbations at large scales and (ii) shift of the granular (non fluid) properties from small to large scales. The latter mechanism finally dominates at all scales and it is responsible for the self-similar characteristics of the clustering.
\end{abstract}

One of the fundamental challenges of modern cosmology is the understanding of the formation of the structures in the Universe. These structures consist of clusters of galaxies and show complex properties extended to very large scales [1]. Usually the simulations and the models aimed at the understanding of these structures are based on three essential elements: (i) the dynamics under the effect of the gravitational forces; (ii) some particular type of initial conditions; (iii) a model for the Hubble expansion [2, 33. In addition simulations are usually run up to a time which is supposed to represent the present state.

Here we would like to take inspiration from these studies and formulate the problem of clustering by gravity in the perspective of the statistical physics of dynamical systems. So we will single out the role of each individual effect at the expense of a loss in realism. We try therefore to identify simple fundamental mechanisms which can be studied in great detail and followed up to their asymptotic state. As a first problem we consider the simple, basic question: how does a random distribution of point masses evolve under gravity? The comparison with the expanding case may then allow us to identify the specific role of this effect. Simulations similar to ours, but in a cosmological context, were performed long ago, e.g., by Itoh et al. [A]. However, we are going to see that the general problematic we consider and the final interpretation will be substantially different.

The main results are: (i) the existence of a well defined thermodynamic limit for the transient regime; (ii) the nature of the clustering process arising from the shift of the granular (non fluid-like) characteristics from small to large scales. (iii) the evolution of correlations shows self similar characteristics.

(c) EDP Sciences 
Self-gravitating systems have been studied in various perspectives since a long time. However, due to the peculiarities of the $1 / r$ potential, most of the concepts and methods of standard statistical physics are problematic [5- 8]. Indeed, the usual approach of statistical mechanics cannot be applied to a system of many point particles interacting by the Newtonian potential, because of (i) the long range nature of the $1 / r$ potential and of (ii) the divergence at the origin [9]. In particular, energy is not extensive, therefore the thermodynamic properties cannot be uniquely defined as the microcanonical and canonical ensembles are not equivalent.

In order to simulate an infinite system, $N$ particles at rest are placed at random into a cube of side $L$ with periodic boundary conditions. Every particle in the simulation box interacts with all the other particles and with the periodic "replicas" of the whole system. The Hamiltonian for a system of $N$ identical particles of mass $m$ is:

$$
H=\sum_{i} \frac{\mathbf{p}_{i}^{2}}{2 m}+\sum_{\langle i j\rangle} m \Phi^{L}\left(\mathbf{r}_{i}-\mathbf{r}_{j}\right),
$$

where the second summation runs over all pairs. The interaction potential which accounts for both the pair contribution and that of the replicas, is:

$$
\Phi^{L}(\mathbf{r})=\sum_{\mathbf{n}}\left[-\frac{G m}{|\mathbf{r}+L \mathbf{n}|}+\int_{V} \frac{G m / L^{3}}{\left|\mathbf{r}^{\prime}+L \mathbf{n}\right|} \mathrm{d} \mathbf{r}^{\prime}\right],
$$

where $G$ is the gravitational constant, $V$ is the box domain and $\mathbf{n}$ runs over all three dimensional vectors with integer components. In the above integral the first term is the usual Newtonian potential due to a particle with mass $m$ placed in the replica specified by $\mathbf{n}$, the second term is introduced to remove the long range divergence of the potential. It can be seen as the potential generated by a repulsive mass $-m$ smeared uniformly over the volume of the same replica. The summation over the replicas is given by the summation of $\mathbf{n}$. The system is individuated by $\mathbf{n}=0$. Such definition for $\Phi^{L}$ gives the expected expression for the force due to an infinite periodic system. Periodic boundary conditions have two major advantages with respect to free boundaries, when simulating an infinite system with a finite N-body representation, namely: (i) they avoid having a preferential point in the simulation and (ii) the total number of particles inside the cube of size $L$ is constant, so particles cannot "evaporate", as would happen in an open system 10]. Furthermore they ensure that both terms in Eq. (11) grow linearly with $N$ for constant density. In order to simulate the evolution of a large number of particles the use of an efficient method for the evaluation of forces is necessary. For our simulations we used a tree-like algorithm of the kind firstly proposed by Barnes et al. [11]. In this algorithm, the force acting on a particle due to its neighbors is evaluated exactly, while that due to distant particles is evaluated by means of a multipolar expansion.

The nature of the gravitational potential requires a time integrator, which is both flexible and efficient. Actually, the short range divergence can produce fast changes in the dynamical variables of a particle, whose motion should be therefore followed with a very short time step. Nevertheless, most of the particles may have a regular and smooth motion which doesn't need such a careful integration. The solution adopted is thus an algorithm, based on a variable and individual time-step. In order to avoid time steps approaching to zero when a very close encounter between two particles occurs, a smoothing in the potential is introduced at very small scales. This, often-used, technique introduces of course an artificial length scale in the simulations. In order to save the Newtonian behavior of the potential, such a scale must be taken much smaller than the average distance between nearest neighbors $\lambda$. In the 
simulations presented here, the smoothing scale is approximately $1 / 32 \lambda$. We have checked on some simulations with $2^{12}$ particles that using a smaller smoothing length does not influence our results; however it slows down even more the code.

The simulations presented in this paper were performed using the tree-code described in 12,13 which we adapted to represent an infinite system by means of periodic boundary conditions according to Ewald prescription (see e.g. 14 $)$.

A small smoothing scale and the absence of cosmological expansion, together with the requirement of high accuracy imply computations heavier than the usual cosmological ones. This is because we consider the generation of structures from a strongly non linear dynamics starting from random initial positions. This will limit the number of particles $N$ in our systems, but all our results will be analyzed with respect to the dependence on $N$. Our simulations are presented using arbitrary units for mass $\left(m_{u}\right)$, length $\left(r_{u}\right)$ and time $\left(t_{u}\right)$. The Newtonian equations depend on a single constant with physical dimensions $(G)$, therefore we can set arbitrarily two of the units and the third is uniquely defined according to $r_{u}^{3} t_{u}^{-2} m_{u}^{-1}=G$.

The left column of fig. 1 shows the projection onto the $x y$-plane of typical snapshots of a system with $N=2^{15}$ particles at different times. All our simulations are performed with a constant number density $n_{0}=N / L^{3}=1 r_{u}^{-3}$. With this choice, a typical crossing time $\tau=\left(m n_{0} G\right)^{-1 / 2}$ corresponds to $1 t_{u}$. On the right column we plot the conditional average density 15] $\Gamma^{*}(r, t)$ of the system at the same time of the corresponding snapshot. $\Gamma^{*}(r, t)$ is the average density in spheres of radius $r$ centered on a particle $i$ of the system. It can be defined as:

$$
\Gamma^{*}(r, t)=\frac{m}{\Omega(r) N} \sum_{i \neq j} \sum_{\mathbf{n}} \theta\left(r-\left|\mathbf{r}_{i}(t)-\mathbf{r}_{j}(t)+L \mathbf{n}\right|\right)
$$

where $\Omega(r)=4 \pi r^{3} / 3$ and $\mathbf{r}_{i}(t), \mathbf{r}_{j}(t)$ are the positions at time $t$ of particles $i$ and $j$ respectively.

In the eq. 3, particles $i$ and $j$ belong to the simulation box, and the summation on the integer vectors $\mathbf{n}$ allow to take into account the replicas of the particle $j$. The three lines in fig. 11 correspond to simulations with $N=2^{9}$ (red), $N=2^{12}$ (blue), $N=2^{15}$ (black). Initial conditions (a) are given by $N$ point masses located randomly and at rest into the simulation box. In the initial phase (b) the system develops clustered structures on small scales. Correspondingly the $\Gamma^{*}(r, t)$ acquires amplitude below the homogeneity scale $r_{0}$, defined by the condition $\Gamma^{*}\left(r_{0}, t\right)=2 n_{0}$. The scale $r_{0}$ can be seen as a typical clustering scale; the reason for such definition will be clearer with the definition of $\bar{\xi}(r, t)$. The red circle in the corresponding snapshot has radius $r_{0}$, and roughly identifies the scale at which the fluctuations in the number density are of the same order of the average value $n_{0}$.

As long as the cluster sizes are much smaller than the size of the box they continue merging and forming bigger and bigger structures (c). Accordingly the homogeneity scale $r_{0}$ increases. Eventually, the typical size of the few remaining aggregates becomes comparable with the box size and they collapse into a single big cluster which contains almost all the matter $(d)$. In order to give a rough estimate the time scale for the formation of the final cluster, we can define a transition time $t_{f}$, such that $r_{0}\left(t_{f}\right)=L / 4$. For simulations with the same mass density, larger $N$ corresponds to larger box size $L$; the final cluster is therefore larger and $t_{f}$ is greater. After the time $t_{f}$ the system reaches a genuine statistically-stationary state. The accuracy of our simulations can be checked by energy conservation. It is a bit tricky to evaluate energy conservation, because in an infinite system there is no absolute value for the potential. Then the relative error in energy $\Delta E / E$ is not a meaningful quantity. For this reason we use the quantity $\Delta E(t) / \Delta K(t)$, the ratio between the error in the total energy and the increase in kinetic energy. Typically, when the final cluster forms the energy conservation is within $1 \%$.

In the first three snapshots of fig. 1 the measured $\Gamma^{*}(r, t)$ function does not depend on 

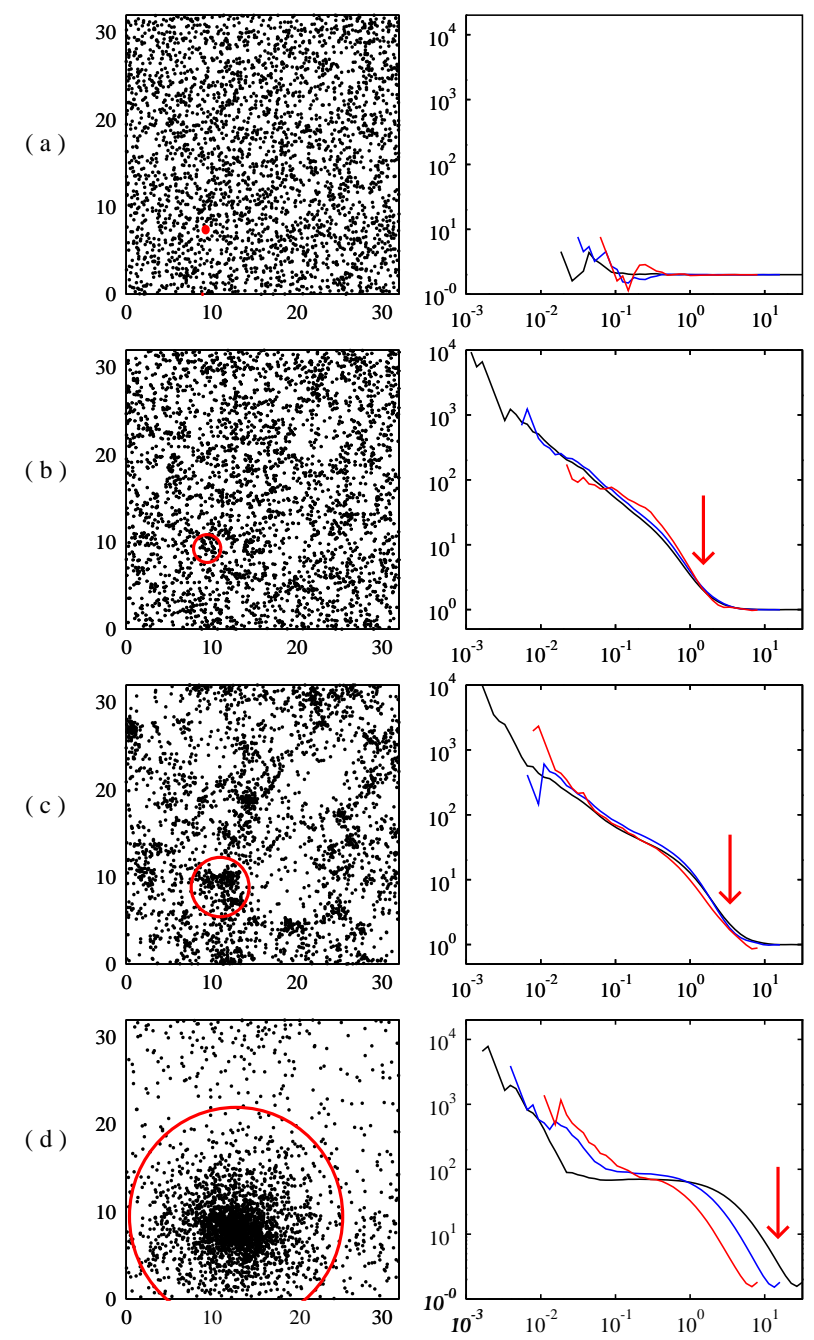

Fig. 1 - Left: projection onto the $x y$-plane at different times of a system with $N=2^{15}$ particles in a simulation box with side $L=32$. (a) $t=0$; (b) $t=0.6$; (c) $t=1$; (d) $t=3.4$. Only $1 / 4$ of the points are shown for the sake of clarity. Right: Conditional average density of systems with $N=2^{9}$ (red), $N=2^{12}$ (blue), $N=2^{15}$ (black). Arrows mark the "homogeneity scale". Note that the structures of the first three figures are size independent, while the size of the system influences the final cluster.

the number $N$ of particles in the simulation box. This is because the characteristic size of the structures is much smaller than the box size. On the other hand, we can see in the last panel (d) that the $\Gamma^{*}(r, t)$ corresponding to systems with different number of particles do not overlap any more. Therefore, the size of the final cluster depends on the box size.

In figure 2 we show the kinetic energy per particle $K(t)$ versus time for the three simulations. We have marked the values of the kinetic energy corresponding to the snapshots in fig. 1. Again, we can distinguish two regimes in the time evolution of $K(t)$ : (i) during the initial collapse phase $K(t)$ show the same growth for the three simulations while (ii) at a later 


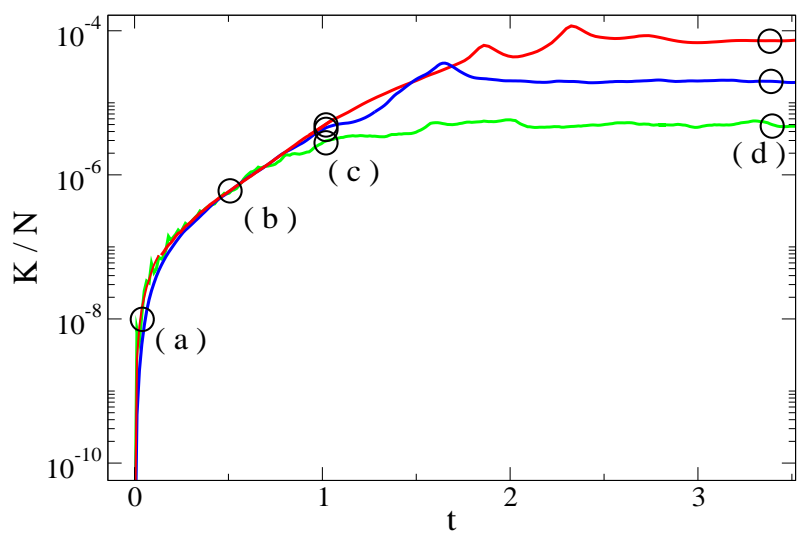

Fig. 2 - Kinetic energy per particle for simulations with $N=2^{9}$ (red), $N=2^{12}$ (blue), $N=2^{15}$ (black). Circles identify the times used in fig. 1. Note that the dynamics of the simulations becomes different only at large times, when the cluster size is comparable to the box size.

time, the equilibrium value depends strongly on the number $N$ of particles. The breakdown of the Gibbs statistical treatment is reflected here by the fact that equilibrium properties of the system, like $K(t \rightarrow \infty)$ and $\Gamma^{*}(r, t \rightarrow \infty)$, do not posses a well-defined thermodynamic limit. In other words, they do not posses a finite limit for $N \rightarrow \infty$ and $L \rightarrow \infty$ with $N / L^{3}$ kept constant. On the other hand, the same quantities appear to be independent on the size of the system for times smaller than $t_{f}$. This suggests that the transient phase, during which collapse occurs, does posses properties with a well behaved limit in the infinite system. This implies that the transient clustering is a well defined statistical properties of the system.

In order to characterize the very small density fluctuations at the largest scales of the simulations, we make use the integrated two-point correlation function of density fluctuations $\bar{\xi}(r, t)=\Gamma^{*}(r, t) / n_{0}-1$. It measures the fluctuations above the average value $n_{0}$ inside a sphere of radius $r$. Our definition of $r_{0}$ then corresponds to $\left|\bar{\xi}\left(r_{0}, t\right)\right|=1$, which is a standard definition for the homogeneity scale 17] In fig. 3 we show the absolute value $|\bar{\xi}(r, t)|$ versus $r$ for the system with $N=2^{15}$. The lowest (black) line represents the correlation function of the initial configuration, which scales as $r^{-3 / 2}$ as expected for points distributed randomly. The other lines corresponds to times $t$ spaced by $1 / 15 t_{u}$. At the largest scales of our simulations, say for $r>10 r_{u}$, the time evolution of the correlation function appears to be well described by the linear approximation for the equation of a self-gravitating fluid [16], which predicts:

$$
\bar{\xi}(r, t)=\bar{\xi}(r, 0) * \cosh ^{2}\left(t / \tau_{l}\right)
$$

with $\tau_{l} \approx\left(4 \pi G \rho_{0}\right)^{-\frac{1}{2}}=1 / \sqrt{4 \pi} t_{u}$. Eq. 目 describes the linear regime in which the time evolution of the system consists solely in an exponential amplification of the initial density fluctuations. Such behavior is indicated in the figure, by the upward arrow labeled by $F$. It is worth noting that in this regime, overdensities increase while underdensities actually decrease; however, in fig. 3 we have plotted the absolute value of $\bar{\xi}(r, t)$, and therefore we see an enhancement of all the fluctuations.

At small scales the dynamics of the system shows completely different properties. For an initial unclustered random distribution the discrete character of points of the system dominates the clustering process at small scales. At the very beginning, i.e. for $t<t_{u} / 4$, the fluctuations grow very fast due to the fall of each particle in the direction of the nearest particle. Actually, 


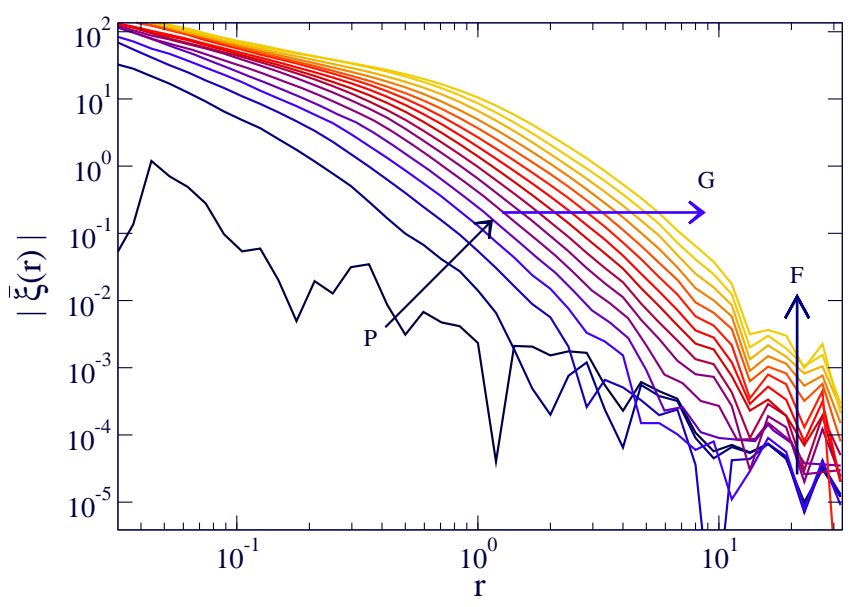

Fig. 3 - Evolution of $|\bar{\xi}(r)|$ between $t=0$ and $t=1$, spaced by time intervals $\Delta t=1 / 15$ for a system with $N=2^{15}$ particles. Arrow $P$ identifies the initial phase dominated by two particles interactions; $F$ is associated with fluid-like evolution at large scales, but the main dynamics for cluster formation is due to the process indicated by $G$, which effectively shifts the granularity from small scales to large scales.

Holtsmark distribution shows that the force acting on a particle in an infinite Poisson system is mainly due to its nearest neighbour. In this phase the dynamics is therefore dominated by two-body interactions. For times greater than approximately $t_{u} / 4$ and at intermediate scales, the $\Gamma^{*}(r, t)$ function satisfies the following equation:

$$
\Gamma^{*}(r, t+\Delta t)=\Gamma^{*}\left(\mathrm{e}^{-\frac{\Delta t}{\tau g}} r, t\right)
$$

with a measured time-scale $\tau_{g}=0.44 t_{u}$, and a similar relation also holds for $\bar{\xi}(r, t)$. This behavior corresponds to a rigid translation along the $r$ axis in a logarithmic plot. This indicates a sort of similarity of structures of different size at different times. Although it is different from the self-similarity as usually intended, it is a clear indication of a sort of scale invariance in the dynamics. Eq. (5) states that the correlations developed by aggregates of size $r$ at time $t+\Delta t$ are the same as those developed by aggregates of size $r \mathrm{e}^{\frac{-\Delta t}{\tau_{g}}}$ at time $t$, and that the time evolution of such correlations is the same.

Visual inspection of the evolution of the system (fig. 1) suggests that clusters formed at a given time are the new discrete elements for the iteration of the clustering process at a larger scale. We propose a simple argument which can account for the behavior described by Eq. (5). Consider a pair of particles at rest with mass $m_{u}$ and at distance $r_{u}$, as in the initial condition of our simulations. Since the leading contribution of the force acting on a particle is mainly due to its nearest neighbor [18], the time scale $\tau_{0}$ for their relative motion is:

$$
\tau_{0}=\sqrt{\frac{r_{u}^{3}}{G m_{u}}} .
$$

After $\Delta t \propto \tau_{0}$ most of the particles are much closer to their nearest neighbor. We will consider such pairs as "bound systems" approximately at rest and distributed randomly with typical distance $r_{1} \approx 2^{1 / 3} r_{u}$ and total mass $m_{1}=2 m_{u}$. We can iterate the same scheme, 
treating the pairs as the new "particles" of the system. The corresponding time scale $\tau_{1}$ for the relative motion is:

$$
\tau_{1}=\sqrt{r_{1}^{3} / G m_{1}}=\sqrt{2 r_{u}^{3} / G 2 m_{u}}=\tau_{0}
$$

Iterating the process we find a sequence of "bound systems" with increasing mass $m_{n}=$ $2^{n} m_{u}$ and length-scale $r_{n}=2^{n / 3} r_{u}$, all with the same time-scale $\tau_{n}=\tau_{0}$. Therefore, being $n \sim t / \tau_{0}$, the scale at which this activity takes place $r(t)$ will obey the following equation:

$$
r(t) \propto 2^{\frac{t}{3 \tau_{0}}} r_{u}
$$

which accounts for the exponential dependence of Eq. (5).

In summary, from the present study we can draw the following conclusions: (i) we address the issue of the cluster formation in a gravity driven dynamics in a system with random initial positions and periodic boundary conditions. Strictly speaking, the system has no well defined thermodynamic limit at asymptotic times. However, it shows a well defined, thermodynamically stable clustering in the transient regime; (ii) we identify the mechanisms of structure formation: initially the system tends to form pairs These pairs then interact with each other and gradually form larger and larger structures, shifting the granular structure from small to large scales. This process appears to be finally dominating over the fluid like dynamics, which tends to amplify the initial large scale fluctuations. These results point to a fundamental importance of the granular mechanism, which appears to lead to a dynamics with elements of self similarity.

To check the validity of our results, we have also repeated some of the simulations with the GADGET code [19].

This work was partially supported by the INFM under the project Clustering and by the EC TMR Research Network under contract ERBFMRXCT960062.

\section{REFERENCES}

[1] F. Sylos Labini, M. Montuori and L. Pietronero, Phys. Rep., 293 (1998) 61.

[2] T. Padmanabhan, Structure formation in the universe (Cambr. Univ. Press, Cambridge) 1993.

[3] W. C. Saslaw, Gravitational clustering in cosmology (Cambr. Univ. Press, Cambridge) 2000.

[4] M. Itoh, S. Inagaki, and W. Saslaw, Ap.J., 331 (1988) 45.

[5] P. Hertel and W. Thirring, Ann. Phys. (N.Y.), 63 (1971) 520.

[6] H. E. Kandrup, Phys. Rep, 63 (1980) 1.

[7] A. Torcini and M. Antoni, Phys. Rev. E, 59 (1999) 2746.

[8] H. J. DE VEGa and N. SANChez, astro-ph/0101568, (2001) .

[9] T. Padmanabhan, Phys. Rep., 188 (1990) 285.

[10] S. Chandrasekhar, Principles of Stellar Dynamics, enl. ed. (Dover, New York) 1960.

[11] J. BARnes and P. Hut, Nature (London), 324 (1986) 446.

[12] P.Miocchi, Ph.D. thesis (Univ. of Roma "La Sapienza") 1998.

[13] R. Dolcetta and P. Miocchi, J. Comp. Phys., 143 (1998) 29.

[14] L.A.Hernquist, F. Bouchet, and Y. Suto, ApJS, 75 (1991) 231.

[15] P. Coleman and L. Pietronero, Phys. Rep., 213 (1992) 311.

[16] J. H. Jeans, Astronomy and Cosmogony (Cambridge University Press, London) 1928.

[17] J. Gaite, A. Domínguez and J. Pérez-Mercader, Ap. J., 522 (1999) L5.

[18] J. Holtsmark, Ann.d.Phys., 58 (1917) 577.

[19] V. Springel, N. Yoshida, and S.D.M.White, New Astronomy, 6 (2001) 79. 